BULLETIN Bulletin hispanique

HISPANIQUE Université Michel de Montaigne Bordeaux

114-2 | 2012

Varia

\title{
Los Vencidos d'Ernest Bark von Schultz
}

roman politique d'un vainqueur méconnu

Dolores Thion Soriano-Mollá

\section{CpenEdition}

Journals

Édition électronique

URL : https://journals.openedition.org/bulletinhispanique/2243

DOI : 10.4000/bulletinhispanique.2243

ISSN : $1775-3821$

Éditeur

Presses universitaires de Bordeaux

\section{Édition imprimée}

Date de publication : 31 décembre 2012

Pagination : 905-917

ISBN : 978-2-86781-855-4

ISSN : 0007-4640

Référence électronique

Dolores Thion Soriano-Mollá, « Los Vencidos d'Ernest Bark von Schultz », Bulletin hispanique [En ligne], 114-2 | 2012, mis en ligne le 05 janvier 2016, consulté le 28 juillet 2022. URL : http://

journals.openedition.org/bulletinhispanique/2243; DOI : https://doi.org/10.4000/bulletinhispanique. 2243 


\title{
Los Vencidos d'Ernest Bark von Schultz, roman politique d'un vainqueur méconnu
}

\author{
Dolores Thion Soriano-Mollá \\ Université de Pau et des Pays de l'Adour
}

Dès son installation en Espagne, en 1884, Ernest Bark von Schultz compose Los Vencidos, roman initiatique et autobiographique. Les perspectives et les tensions que le paradigme vainqueur-vaincu sous-tend sont explorées par l'auteur dans cet exercice de création littéraire mis au service d'une réforme sociale-régénération-de l'Espagne et de la création d'une Union européenne avant la lettre.

Mots-clés: Bark Von Schultz (Ernest), Los Vencidos, roman politique, autobiographie, régénérationnisme.

Desde su llegada a España, en 1884, Ernesto Bark von Schultz compuso Los Vencidos, una novela iniciática y autobiográfica. En este ejercicio de creación literaria el autor explora las pespectivas y tensiones que el paradigma vencedor-vencido sustenta para ponerlo al servicio de la reforma social -o regeneración- de España y de la creación de una Unión europea avant la lettre.

Palabras claves: Bark Von Schultz (Ernest), Los Vencidos, novela política, autobiografía, regeneracionismo.

As soon as he settled in Spain, in 1884, Ernest Bark von Schultz wrote "Los Vencidos", an initiatory and autobiographical novel. The underlying perspectives and tensions within the "winner-defeated" paradigm are explored by the author, in his exercise of literary creation to the service of social reforms -or regeneration - in Spain and the creation of an European Union before its very existence.

Keywords: Bark Von Schultz (Ernest), Los Vencidos, political novel, autobiography, regenerationism.

$\mathrm{N}$ ombre de grands hommes, consacrés par la notoriété publique, abondent dans les Histoires officielles. D’autres, cependant, dotés de considérable stature, tel Ernest Bark von Schultz (Kaava, Estonie, 1853-Madrid, 1922), qui ont tranché sur leurs contemporains et par leur existence et par leur pensée, 
sont condamnés au silence dans ce qu'il y a lieu d'appeler le récit historique. Pourtant la présence et la promptitude d'esprit d'Ernest Bark se sont répandues de la Russie des Tsars à l'Espagne de la Restauration et ont immanquablement contribué à secouer l'autorité établie, l'oppression ambiante, l'ankylose des préjugés et à transformer un certain nombre de pratiques ressortissant aux protocoles politiques, sociaux, voire esthétiques. Dans notre étude, nous entendons retracer son histoire afin de mettre en évidence les décalages que Bark instaure entre son propre vécu et ses représentations, lorsqu'il mêle étroitement son histoire à l'Histoire de l'Europe de la fin du XIX ${ }^{\mathrm{e}}$ siècle et du début du XXe.

Lors de son installation en Espagne, en 1884, Ernest Bark compose un roman intitulé Los Vencidos. Il y retrace les péripéties de son périple personnel, y dépeint les événements politiques qui l'ont vu naître ou les contextes historiques qui l'ont déterminé. Peut-être pourrions-nous dire que dans Los Vencidos, la littérature puise dans l'Histoire, une histoire anonyme car individuelle et méconnue. Il faudrait également ajouter qu'à l'inverse l'Histoire fait l'emprunt du récit littéraire pour se raconter de manière vivante, voire poétique. Emprunts que Bark opère, peut-être, de manière plus instinctive que théorisée, dans le but de s'interroger lui-même, de questionner son imaginaire, ses engagements socio-politiques et l'ensemble des réalités les plus porteuses de souffrances. Notre objectif n'est pas ici de réfléchir sur le statut du récit littéraire dans l'Histoire et pour l'Histoire, mais simplement sur le paradigme vaincu/vainqueur dans le roman d'Ernest Bark, modèle d'interculturalité à la fois représentatif et singulier de la fin du XIX ${ }^{\mathrm{e}}$ siècle. En Europe, Ernest Bark est un passeur d'idées, un propagandiste au service de la Révolution Sociale dans le contexte " régénérationniste " espagnol précédant la fin de l'Empire, au moment même où le sentiment de la décadence commence à agiter les intellectuels et les artistes. Dans le cadre que lui offre la fiction, laquelle par le biais du récit littéraire œuvre au travail de mémoire, il nous sera loisible de découvrir sa trajectoire personnelle : vaincu en Russie, il est paradoxalement vainqueur dans son engagement personnel et, sans conteste, vainqueur dans sa vie amoureuse.

\section{I. - Ernest Bark, un Estonien en Espagne}

Issu d'une famille de la petite aristocratie balto-allemande, Ernest Bark von Schultz est un jeune rebelle. Étudiant en Économie et Commerce à l'École Polytechnique de Riga, il délaisse ses études pour participer très activement au Mouvement du Réveil de l'Estonie et faire aboutir ses revendications contre l'oligarchie. Ces mêmes revendications le poussent à prendre part à la guerre contre la Turquie (1877) car il croit fermement au renversement du pouvoir du tsar et à l'instauration de la démocratie par les militaires. Il déserte et poursuit ses études en Histoire, en Philosophie et en Politique aux universités de Leipzig, Munich et Berlin et ce jusqu'en 1881. Ses contacts avec les 
nouvelles sciences - la sociologie et la psychologie - ainsi qu'avec les doctrines et pratiques politiques de l'époque, notamment le marxisme, le Kulturkampf et l'Impérialisme allemand, sont décisifs dans la formation de sa pensée. Afin de se consacrer à l'étude de la psychologie des peuples, il dilapide la fortune qu'il a reçue en héritage lors de nombreux voyages en Europe. En Suisse, où voit le jour Der Baltische Föderalist (1883), le premier journal clandestin qu'il fonde, il reprend contact avec les anarchistes et les fédéralistes baltes exilés. Le succès du journal le conduira au bagne en Sibérie.

À la fin de 1884 Ernest Bark arrive en Espagne afin de retrouver Matilde Cabello, une jeune Andalouse qu'il avait rencontrée lors d'un premier voyage en ce pays. Elle deviendra son épouse et la mère de ses six enfants. À Madrid, Ernest Bark travaille comme correspondant pour les journaux Köln Zeitung et le National Zeitung de Berlin, avant de fonder des journaux internationaux en langue allemande, tel que l'hebdomadaire Deutsche-Spanisch Revue (1886), Spanien (1898-1899), puis Correspondance latine (1890).

Il intègre sans peine les cercles des républicains radicaux, des journalistes, des bohèmes progressistes, et il fonde le Parti Démocrate Socialiste, la Agrupación Demócrata Social, et le groupe Germinal, ainsi que leurs relais médiatiques tels que El Crisol (1890-93) à Alicante et Cartagena Moderna (1893-1895), ou encore, des périodiques plus ambitieux comme La Democracia Social (1890 et 1895) et Germinal (1897-1903). Ces journaux sont tous animés par le même esprit progressiste et républicain qui caractérise les autres organes de presse dans lesquels Ernest Bark publie des articles : El Pais, Las Dominicales del Librepensamiento, El Radical, La Piqueta, Nuestro Tiempo, El Mundo, etc. Le large éventail de sujets socio-politiques et culturels qu'il propose dans ces colonnes de presse vise à la divulgation d'idées progressistes et cosmopolites. Par ailleurs, au sein du Républicanisme radical, Bark retrouve le cadre propice à la mise en place de son projet de réforme socio-politique en Espagne. Il propose une République Sociale moderne et scientifique appelée à s'ériger en modèle politique d'ordre universel. De même, son programme de réformes se structure autour des secteurs économiques et technologiques, nécessaires au progrès matériel. Quant au progrès humaniste, il se fonde sur l'évolution des valeurs philosophiques ainsi que sur la réforme éducative et culturelle au service d'un Socialisme Positif inspiré du socialisme humaniste français, et notamment de Proudhon.

\section{II. - LES VAINCUS, ROMAN AUTOBIOGRAPHIQUE}

En 1890, après six ans de résidence en Espagne, Ernest Bark a la hardiesse - ou le courage - de se lancer dans la création littéraire en langue espagnole. Le roman Los Vencidos signifie pour lui une vraie aventure littéraire et ce, compte tenu de sa faible maîtrise de la langue en $1891^{1}$. Il le conçoit comme le symbole

1. D'abord sous la forme d'un feuilleton dans le quotidien El Liberal (juillet-août 1891, Alicante), puis sous forme d'ouvrage : Bark, Ernest, Los Vencidos, Alicante, Impta. El Liberal, 
de la paix intérieure qu'il parvient à profondément ressentir. D'une part, il retrouve une vie paisible après un itinéraire vital mouvementé et les persécutions que lui a infligées le gouvernement espagnol consécutivement à ses critiques politiques dans la presse allemande ; d'autre part, la prise de parole au moyen d'un roman politique lui permet de solder la dette qu'il ressent à l'égard des circonstances historiques, de son passé, de son présent et de se projeter dans l'avenir pour l'avènement de la Modernité2.

Les cercles libres-penseurs et intellectuels, fortement impliqués dans les mouvements progressistes, ont accueilli avec curiosité l'activisme politique de Bark. En étroite interaction avec son contexte, son roman jouit d'un grand succès. Les dimensions historiques et modernes de Los Vencidos, "un verdadero compendio de socialismo internacional y una historia verdadera del movimiento revolucionario ruso " (El Crisol, 19-07-1891), sont chaleureusement applaudies. Toutefois, les louanges de la presse semblent quelque peu excessives. Los Vencidos n'est point un résumé historique des premières organisations activistes à l'origine de la Révolution russe mais la narration des avatars de la vie d'un personnage qui se pose lui-même, au plan de la fiction, en modèle de lutte sociale. De fait, le récit se présente comme un outil privilégié de compréhension de son parcours existentiel mais aussi comme un vecteur de changement : il est porteur d'effets à la fois sur le narrateur et sur ses destinataires (présents ou non), d'autant plus que, sous prétexte d'écrire un roman réaliste socialiste, Bark offre une version romanesque de sa pensée politique, philosophique et sociale. Même si dans Los Vencidos, la parole littéraire véhicule des expériences vécues dans le but de témoigner en tant que vaincu de l'Histoire, il n'en demeure pas moins que la parole littéraire est également porteuse d'espoir. Elle dit l'histoire d'un vaincu mais également le devenir d'un vainqueur.

Puisque sa biographie en est la source d'inspiration elle devrait rendre aisée la composition du roman. Cependant, dans Los Vencidos, il est des stéréotypes littéraires et des modèles romanesques de facture connue. Médiocrité artistique et manque d'originalité sont tangiblement présents. À l'écriture facile se joint l'appel à la simple reconnaissance des lecteurs. Le but de Bark n'est pas de les surprendre sur le plan esthétique : il table sur l'émotion et l'idéologie. Autrement dit, il vise à convaincre le lecteur de participer à la réforme sociale, puis à affirmer la mission prosélyte de l'écrivain et le rôle de la littérature dans la société, s'inspirant du romantisme idéaliste auquel il adhère. Il conjugue ainsi le récit autobiographique avec le roman d'éducation (Bildungsroman), et il y ajoute des stratégies propres au roman historique d'aventures.

$\mathrm{Au} \mathrm{XIX}^{\mathrm{e}}$ le récit autobiographique faisait partie des traditions littéraires allemande, anglaise et française ${ }^{3}$. En Espagne, comme le disait Emilia Pardo

1891, réédité par Dolores Thion Soriano-Mollá, Alicante, Instituto de Cultura Juan GilAlbert, 2005. Toutes nos citations proviennent de cette dernière édition. Également : RIOS CARRATALA, Juan Antonio, «La novela de un radical, Ernesto Bark», Homenaje a Antonio Vilanova, Barcelona Universidad, 1989, p. 557-566.

2. Ibid., p. 127.

3. Très présent dans les milieux protestants, l'exercice de l'écriture intime était vivement 
Bazán, la biographie était contraire aux goûts nationaux : soit qu' elle fût refusée par une modestie réelle, soit qu'elle fût proscrite par un orgueil démesuré ${ }^{4}$. Ortega y Gasset en convenait, lui aussi, qui précisait à son tour que, dans son essence, la race espagnole et son manque de volupté vitale y étaient pour beaucoup 5 . Pour sa part, Ernest Bark masque la nature autobiographique de son roman en recourant à l'artifice du récit «trouvé». Comme dans le Quichotte ou dans le roman romantique d'aventures, le narrateur transcrit fidèlement une histoire qui lui a été confiée ${ }^{6}$. Une histoire dont il se croit obligé de certifier l'authenticité ainsi que la fidélité du récit rapporté. Nulle intention de se dérober fictivement à la responsabilité de l'œuvre ou aux préjudices qu'elle pourrait lui attirer en tant qu'étranger, car l'artifice rhétorique du chroniqueur sincère, objectif et neutre, garantit l'historicité du texte et insère les sources de l'histoire personnelle dans la prestigieuse tradition écrite. À la différence du roman romantique, le récit de Los Vencidos se construit sur un très léger anachronisme, dans un passé relativement proche, permettant au narrateur de recréer sa propre rétrospective. Les éléments tragiques nécessaires au maintien du suspense et à la progression de l'action sont annoncés dès les premières pages, instaurant ainsi un jeu efficace d'analepses et de prolepses, mais surtout des effets de réalisme.

Malgré la nature présumée fictive du protagoniste, Bark affuble Erico Orloff, son alter ego, de ses propres attributs, de sorte que le lecteur peut sans peine aucune reconnaître les modèles qui sont à la source de son inspiration :

El era alto, delgado, con bigote rojizo y pelo del mismo color encrespado y ojos azules de felino; ella era baja, algo gruesa, graciosa de aspecto. Cuando salian los dos a pasear cogidos del brazo se extrañaba la gente del contraste de la pareja?.

Autant l'écrivain que son double fictionnel sont à l'image de Neptune à l'air colérique $^{8}$; ce sont des êtres hallucinés et étranges, rêveurs, au caractère plus méridional que nordique, des hommes impulsifs, fougueux mais sincères et adeptes de la polémique comme thérapie régénératrice. La fidélité du personnage littéraire est presque absolue. Les événements, les faits, les personnes, les données sur la biographie de Bark sont incorporés à la fiction dans la plus stricte rigueur chronologique, spatiale et historique, rapportés selon une relation de causalité dans un temps linéaire.

Parce que Bark met en place tous les dispositifs pour créer un effet de réalité, ce récit de la vie de Bark se présente comme une co-construction du sens. De

recommandé pour l'éducation morale, notamment féminine, du fait de s'appuyer sur l'examen des consciences et la confession.

4. Pardo Bazán, Emilia, Apuntes autobiográficos, in Kirby, Jr. Harry L., OOCC, III, Madrid, Aguilar, 1973, p. 725.

5. Ortega y Gasset, José, La deshumanización en el arte e Ideas sobre la novela, Madrid, Revista de Occidente, 1925.

6. Bark, Ernest, op. cit. p. 127.

7. Vallina, Pedro, Mis memorias., México, Caracas ed., 1968, p. 63.

8. Bark, Ernest, op. cit. p. 150. 
même, elle se présente comme la voie privilégiée pour la compréhension des enjeux sociaux et politiques de l'Europe de son temps. Le récit rétrospectif requiert l'auto-représentation des événements de la vie que Bark décide de rapporter. Nous assistons à une double construction du discours politique du roman : celle de la perception des événements supposant une première interprétation, et, en même temps, celle de la composition du récit romanesque qui les recrée. En effet, il accommode l'histoire qu'il vise à faire connaître en fonction des points de vue d'un discours de vaincu.

Le discours du vaincu s'établit dans un rapport dialectique avec le discours officiel et s'affirme en s'opposant à lui. Cependant, Bark ne propose pas une nouvelle vision qui contredirait l'histoire officielle. C'est en la revisitant qu'il en dénonce les fourvoiements. Il désavoue l'abus de pouvoir, les rapports de force entre les nations, puis les vices des hommes d'État, des monarchies, des institutions religieuses, des races et des peuples. Par de courtes synthèses, des énumérations de lieux ou des citations de personnages historiques glissées dans les monologues intérieurs d'Erico Orloff, par les interventions du narrateur ponctuant de jugements et d'exégèses la pensée du protagoniste, Bark dévoile à quel point se perpétuent depuis le Moyen Âge les notions d'autoritarisme, de népotisme, d'exploitation, d'ambition, de cupidité et tous les autres vices de la nature humaine, lesquels rendent impossibles la liberté et la paix parmi les hommes. Ainsi, ses reproches de vaincu en ressortent-ils légitimés par leur inscription dans la tradition historique de l'Europe. Bark en est conscient. Le passé permet d'expliquer le présent et de projeter l'avenir. Un avenir qu'il augure nouveau grâce au changement de rôles, des vaincus en vainqueurs, des vainqueurs en vaincus.

Il va sans dire qu'entre fiction et réalité il existe toujours un espace que l'auteur manipule en fonction de ses intérêts, de ses intentions et de ses objectifs. A l'image de Bark, Orloff est l'aventurier, le héros de la cause sociopolitique russe, capable de tout sacrifier au service du nihilisme. À l'image de Bark, Orloff avait connu Matilde, la jeune fille idéalisée à partir des modèles des vierges du Titien et de Murillo, la femme qu'il épouserait à la fin heureuse de son roman. La problématique du roman se fonde sur la lutte politique et le conflit intime, la dialectique entre vainqueur et vaincu à la fois dans l'Histoire et dans l'histoire amoureuse du protagoniste.

Sur ces deux plans, le jeune Orloff se débat tout au long du récit puisqu'il retrace le processus d'acquisition de la maturité et de la refonte personnelle des idées apprises. S'inspirant du roman d'éducation allemand (Bildungsroman), ce processus prend les formes symboliques du voyage, un voyage initiatique qui modèle la personnalité et qui lui offre l'opportunité de construire expérimentalement sa propre conception du monde et d'orienter sa trajectoire au contact de la vie. Par là même, le voyage constitue un tout qui donne sens aux événements rapportés, les organise et détermine l'ordonnancement du roman.

Après de longues années de flânerie à travers l'Europe, le voyage initiatique s'amorce et s'achève en Espagne, au point même du départ, afin d'imprimer à la vie l'image circulaire du temps moderne. La cause première de son voyage 
relève de l'impossible : le dessein de Bark est de rentrer dans son pays d'origine, d'abandonner l'Espagne pour la Russie. Obligé de quitter cette dernière au péril de sa vie, il l'a remplacée par un pays d'adoption, l'Espagne. Et pour lors, il se permet de faire le chemin inverse. Dans ce va-et-vient, la Suisse et l'Autriche vont jouer le rôle d'intermédiaires. Ce sont avant tout les espaces où il exerce son métier de journaliste au service de la révolution socialiste aussi, semblable périple revêt-il essentiellement un caractère politique. Toutefois Bark a soin de ne point totalement oblitérer ni la part ressortissant à la topographie ni celle dévolue à l'intimité. Chaque étape est un fragment de réalité dont Bark doit rendre compte pour lui faire prendre sens, ce qui requiert un travail à la fois d'apprentissage et de mémoire.

Chaque pays sert de cadre référentiel à de nouvelles expériences politiques, professionnelles et amoureuses, à un état de crise dans la solitude la plus complète, mais également à une initiation jouissant du soutien de personnages bienfaiteurs, toujours secondaires. Orloff progresse ainsi dans son développement personnel. Muni de ses idéaux politiques, dévoré par un feu passionnel, il surmonte des épreuves, franchit maints obstacles toujours au risque de sa propre vie. Il incarne l'activiste politique, le bohème aventurier et engagé qui rêve d'une vie "en conjunto tan artística y bella como una hermosa obra de arte, una novela de verdad que tuviese páginas sentimentales y trágicas y siempre verdades interesantes " ${ }^{9}$. Tout au long de son parcours initiatique, les intrigues relèvent du roman gothique : des prophéties, des persécutions, des vengeances, des origines nobles voilées, des enfants illégitimes. Médiums, fausses identités, vols et incendies, configurent l'architecture romanesque du roman. Parce que Los Vencidos est d'abord paru sous la forme d'un roman-feuilleton, ces stratégies littéraires des romans à succès du XIX ${ }^{\mathrm{e}}$ concourent avec grande efficacité à la création d'un public lecteur fidèle et, en particulier, à la diffusion des idéologies politiques progressistes.

La trame amoureuse, conforme au goût des lecteurs de l'époque, sert également d'artifice pour véhiculer la pensée politique. Ainsi, est-elle retenue au premier plan aux moments-clefs de l'intrigue, comme l'est, par exemple, le début du roman. A Málaga, en effet, le 6 juin 1882, Erico s'éprend d'une jeune femme, Matilde, personnifiant «la feliz Andalucia, tan admirada por los pueblos del Norte " ${ }^{10}$. Mais Orloff se voit dans l'obligation de choisir : amour ou lutte anti-tsariste. Bien sûr, la lutte anti-tsariste l'emporte. À ses yeux, renoncer à ses idées reviendrait à se trahir soi-même. Comment conjuguer l'amour avec son engagement alors que :

En Rusia aún está muy lejos la victoria. La vida del revolucionario ruso, o sea, del nibilista como le llamaron los interesados defensores del despotismo para desprestigiarle, es una cadena de sacrificios continuos y amarguras cuyo fin suele ser la triste emigración al extranjero, la más triste y desconsoladora abdicación de los ideales o la muerte en los calabozos, en Siberia o en el cadalso ${ }^{11}$.

9. Bark, Ernest, op. cit., p. 262.

10. Ibid., p. 130-131.

11. Ibid., p. 142. 
L'altruisme d'Erico rencontre ainsi ses failles face au mystère de l'amour dans un conflit intérieur qui fragmente la conscience du protagoniste. Ayant sacrifié son existence au mouvement révolutionnaire anti-tsariste, sa vie en exil se résume à un véritable chemin de croix et de souffrance.

Conformément à l'itinéraire de Bark, le roman s'organise en trois parties calquées sur les déplacements déjà cités : d'abord, de l'Espagne en Russie, en passant par la Suisse, ensuite, l'Autriche et enfin, le retour en Espagne, avec une courte étape en Italie.

Vaincu, car exilé et écarté du champ d'action directe, vaincu parce qu'il refuse l'amour, Erico Orloff inaugure son parcours dans le voyage qui le conduit de l'Espagne à Genève, centre du nihilisme russe. Il ne lui reste que le droit à la parole et la possibilité de circonvenir les autorités en diffusant un périodique clandestin. Il s'agit de la revue en langue allemande Der Baltische fóderalist (1882), revue pamphlétaire anti-tsariste visant également à la diffusion du socialisme international. Malgré son nationalisme, Der Baltische föderalist se présente comme le porte-parole de tous les peuples opprimés, en accord avec la nature cosmopolite et universelle que Bark attribue au journalisme. Réformiste modéré, Bark - ainsi que son alter ego Erico - propose un programme de restructuration fédéraliste, démocratique et pacifique de la Russie.

La capitale autrichienne constitue la deuxième étape de ce voyage, marquée par le désenchantement du journaliste, mais émaillée de nouvelles figures féminines : l'autrichienne Leopoldina et la russe Olga Ivanovna. Deux types de femmes, l'une ignare et naïve, l'autre intelligente, libre et révolutionnaire, qu'Orloff érige en métaphore de la mère Russie. Dans la suite de l'intrigue, le faux ménage, composé d'Olga et d'Erico, voyage en Russie où se préparent des attentats contre le Tsar. Le moment venu, ils se doivent d'organiser la suite du mouvement socio-politique. Poursuivi et menacé, Erico est obligé de se cacher à Vienne, où il éprouve une fois de plus des sentiments de frustration et de solitude. Dès lors, Olga représente l'ange gardien d'Erico, lui prodiguant ses conseils lorsqu’à chaque étape du voyage surviennent ses crises :

Adiós Erico, lo envidio por su idealismo,... usted ama de verdad a mi desgraciada nación y a Rusia, pero no está llamado a sacrificarse por ella, porque tiene usted otra misión que cumplir. Llamar y despertar a los pueblos libres de Europa y América para que acaben su obra de redención, resolviendo el gran problema social, esto es su deber y esto es la única salvación de Rusia, porque el absolutismo solo caerá bajo la presión de la libre Europa, y, entonces, será redimido nuestro pobre campesino y mi nación renacerá a otra vida ${ }^{12}$.

Si jusqu’à présent, en tant que vaincu, Bark croyait sa destinée fatalement déterminée par le devoir impératif d'offrir la liberté au peuple russe, dorénavant la frustration du révolutionnaire brise le difficile équilibre entre le sacrifice pour la cause anti-tsariste et sa volontaire privation affective. Dans la détresse, Erico, sombrant dans un pessimisme schopenhauerien, perd la volonté de vivre, une volonté qui le dotait d'une "fuerza titánica » dans la lutte et dans son existence

12. Ibid., p. 169. 
de nihiliste émigré, ce qui le décide à suivre les conseils de changement de vie proposés par sa bienfaitrice, Olga Ivanovna.

Le tournant nécessaire marquant la transition vers la deuxième étape du voyage s'opère subitement par l'arrivée d'une lettre de Málaga. À Vienne, le protagoniste trouve une certaine stabilité financière qui lui permet d'envisager et de financer son installation en Espagne. C'est alors que commence la transformation du vaincu en vainqueur. Nous assistons au processus de transformation puisque le roman se veut une fresque psychologique, de " las revoluciones de los caracteres y de las ideas ". Ne maîtrisant pas l'introspection analytique, le narrateur se documente sur les spéculations philosophiques et historiques nécessaires à un tel changement. Notre scénario dispose maintenant d'une toile de fond pangermanique avec des objectifs plus accessibles : la réforme sociale en Europe, pour laquelle il retient l'Espagne qui, selon lui, présente une conjoncture favorable.

L'activisme d'Orloff à Vienne se matérialise dans les journaux Deutsche Zeitung et Wiener Revue, ce dernier étant fondé dans le but de diffuser un socialisme réformiste :

Quien nunca ha sentido latir en su pecho el santo fuego del entusiasmo por el apostolado de una idea, no puede comprender el goce que experimentaba el convencido socialista en trabajar desde su publicación quincenal por la realización de sus ideales ${ }^{13}$.

Orloff est soudainement baigné de romantisme ; sa souffrance déclenche une problématique nouvelle sur laquelle réfléchir : "iSi la libertad, el progreso, no es la felicidad!... ;Ah, difícil problema, secreto eterno! ¿Qué es la felicidad? " ${ }^{14}$. Il trouvera sa réponse : son propre accomplissement dans l'amour. Toutefois, c'est la liberté de parole dans la presse, ou la littérature, qui lui permettent de vaincre son exil intérieur et extérieur. En outre, la parole reste l'instrument principal de la lutte dans l'opposition, l'instrument de construction de la contre-histoire et, encore, l'instrument pour divulguer de nouvelles idées, de nouveaux espoirs de changement. Pour Ernest Bark, les Lettres représentent l'expression culturelle et artistique de l'humanité, un moyen au service de l'éducation, de la sensibilité et de la transmission idéologique pour la construction d'une République Sociale d'ordre universel. Lettres de vaincu, certes, mais non défaitistes. Bien au contraire, elles se doivent d'insuffler la force et l'espoir de la reconstruction. C'est dans ces termes que le jeune protagoniste parvient à surmonter les obstacles tout au long de son voyage initiatique et décide de son retour en Espagne, ceci non sans la recherche d'effet :

Una voz interna le decía que la bella nibilista tenía razón, que estaban vencidos, que no habia esperanza alguna sino la que les anunciaba la alborada del nuevo sol de la humanidad en los horizontes de la revolución social que el conocedor de toda aquella Europa del Norte petrificada en preocupaciones de castas y razas no vio levantarse ni desde Alemania, ni desde Francia, sino desde las crispadas peñas de las sierras de España... ${ }^{15}$.

13. Ibid., p. 193.

14. Ibid., p. 198.

15. Ibid., p. 214. 
L'aventure est la caractéristique singulière de la troisième étape du voyage. Le dédoublement de perspectives, partagées entre Matilde et Erico, insuffle du dynamisme au récit durant le processus d'intégration du protagoniste à la vie madrilène. Ses objectifs se sont précisés : il assume son statut de vaincu en Russie, tout en se flattant d'être un vainqueur dans sa soif de "el ideal sublime del socialismo internacional", lequel aurait tôt ou tard des retombées bénéfiques sur les causes perdues de son peuple. Même s'il connaît encore des situations de détresse à Madrid, centre névralgique du "vicio, del dinero, del cálculo $»^{16}$, même s'il supporte tant de gêne financière et endure l'hostilité de l'oligarchie des caciques et du clergé, l'intolérance et la décadence, il refuse :

Declararse vencido también aqui, hubiera sido el suicidio intelectual y moral. Erico protestaba con toda la energía de su alma contra esta muerte, porque habia venido a España huyendo de la petrificación que le amenazaba convirtiéndole en una rueda de máquina de la prensa de Viena y buscando un punto de apoyo para continuar su lucha por la libertad en Rusia y la redención social de su patria y Europa entera ${ }^{17}$.

Son expérience de journaliste lui offrant la possibilité de se frayer un chemin dans le maquis de la presse espagnole et de pourvoir à ses besoins financiers, Orloff pourra non sans mal retrouver sa bien-aimée en la soustrayant à temps aux griffes de l'usurier Don Carlos. Les parents de Matilde en avaient, en effet, marchandé le prix auprès de ce dernier en raison des avantages financiers que le mariage aurait pu leur procurer. La victoire d'Orloff dans le duel qui l'oppose à don Carlos clôture le périple initiatique. Non seulement Orloff a atteint sa maturité, mais encore, sa victoire publique en amour, qui parachève sa transformation en personnage de vainqueur. Bark nous apparaît comme n'ayant pas livré autre chose qu'un de ces combats intérieurs par lesquels l'homme lutte contre lui-même pour se contraindre à plus de dignité, de noblesse et de perfection. Vaincu en politique, vainqueur en amour, il sera finalement vaincu, puisque Matilde réussira à convertir Orloff, le panthéiste, au catholicisme afin de légaliser leur amour.

\section{III. - L'ESPOIR D'UN VAINCU, VAINQUEUR}

Il est frappant de constater que la personnalité du vaincu relève d'une psychologie forte. Jusqu'à présent nous avons repris les qualificatifs qu'il s'approprie tout au long du roman. Or, nous l'avons déjà précisé, s'agissant d'un roman de formation, d'errance initiatique, où la politique et l'amour constituent les deux vecteurs conduisant le protagoniste à sa réussite, à son perfectionnement et au bonheur, notre vaincu portait en réalité en soi un vainqueur en puissance. Le voyage ne sert qu'à faire ressortir ses potentialités. Ainsi en tire-t-il sa morale: "La desgracia enaltece y purifica a los buenos y fuertes,

16. Ibid., p. 238-239.

17. Ibid., p. 231. 
a los débiles arroja al fango o les hace desalmados y crueles, y los malos se pierden en el abismo del crimen " ${ }^{18}$.

À chaque moment de l'histoire le vainqueur et le vaincu peuvent curieusement partager les mêmes traits de supériorité. Du reste, ce n'est qu'une question de perspective. Durant les périodes d'agitation sociale, de crise des systèmes politiques et des nations, le vaincu engagé incarne l'opposition et la dissidence. Pour lui-même et ses pairs il est un esprit d'une grande valeur morale eu égard à ses sacrifices altruistes, alors que les vainqueurs en font un brigand car il représente une menace pour le pouvoir. Ce sont les rapports de pouvoir (ou l'abus du pouvoir au nom du tsar, de la monarchie, du parlementaire corrompu ou du cacique) qui creusent les distances entre les hommes, vainqueurs ou vaincus. Reste l'espoir que toute modification de la conjoncture devrait faire perdre ce qui a été gagné ou gagner ce qui a été perdu. Mais l'épée de Damoclès reste, elle aussi, toujours suspendue.

Ce mouvement, dans la fiction, qui mène du vainqueur au vaincu, engendre de nouvelles formes littéraires dans le texte en faisant évoluer les genres et, notamment, en dépassant le modèle du héros. Puisque Erico Orloff est conçu à l'image d'un héros romantique, il est le symbole de l'idéalisme, mais aussi de l'échec et de la frustration. Il est un personnage qui se sait destiné aux Lettres et à la politique depuis son enfance, mais son déracinement dans l'exil le réduisant à faire partie d'une minorité étrangère condamne d'avance sa vie professionnelle. Toutefois Bark oblige son personnage à dépasser le modèle littéraire grâce à sa force personnelle. Véhément et combatif, Ernest Bark - ou Orloff - se pose, alors, comme le modèle de "la abnegación altruista ", du citoyen engagé au service de la société. Dans l'accomplissement de son devoir salutaire, Orloff le vaincu symbolise les valeurs républicaines universelles - la justice, la liberté, l'égalité, la fraternité -, les vainqueurs étant l'emblème de l'oligarchie, de l'avilissement ou de la méprisable dégénérescence - portrait, par exemple qu'Ernest Bark dresse tant des tsars que des Bourbons parce que leur pouvoir réside dans l'exercice d'une autorité tyrannique, particulièrement dans le cas des premiers. À cause de ses engagements civiques l'écrivain ne se renferme point dans les limites de la vie intime et individuelle de ses créatures. Bien au contraire, il met au premier plan les conflits historiques au détriment des conflits individuels de sorte que le devenir de chaque créature est le fruit ou la conséquence d'une conjoncture historique. Cette subordination au monde extérieur rapproche Los Vencidos des mémoires et renouvelle le genre littéraire autobiographique :

Nunca habia mirado Erico tan profundamente en los horribles abismos de nuestra sociedad, en este caos de anomalias absurdas, injusticias irritantes y abandonos criminales. Al experimentar en su propia desgracia los defectos del orden social presente, se afirmaba más aún en sus entusiasmos por los ideales del porvenir ${ }^{19}$. 
Le changement de contexte ou de mission dans la vie permet au protagoniste de retourner sa position de vaincu en vainqueur. Ainsi, sa frustration à l'égard de son pays, pourra-t-elle disparaître et son bonheur personnel et ses engagements humanitaires pourront-ils être satisfaits. L'Espagne en garantit la transformation :

Quizás esté llamada la bella España a ponerse otra vez al frente de este gran movimiento civilizador; tras largo sueño ha despertado a nuevos ideales y puede guiar a la vieja Europa al nuevo mundo de la idea social del porvenir, como ya una vez le abrió los horizontes de otro mundo nuevo ignorado ${ }^{20}$.

Même si dans ce "nuevo mundo desconocido " la permutation des rôles ne s'effectue que par la force déterminante du contexte, ce sont précisément l'état critique de l'Espagne de l'époque et les singularités de l'âme nationale qui, en outre, en favorisent la perspective. En Espagne, Erico partage le sentiment de crise, les pathologies que les intellectuels décèlent dans cette société. Ses «sombras negras" légitiment sa nouvelle mission:

[...] Con su mirada penetrante: sobre todo aquella sociedad ficticia, vacia, apercibia pronto una espesa red que todo lo envolvía y desde Madrid se extendía sobre el desgraciado país, impidiendo todo desarrollo libre e independiente y empozoñado toda la vida nueva y honrada que pudiera brotar ${ }^{21}$.

Dans ce déterminisme contextuel, le non-conformisme d'Ernest Bark, sa ténacité, son désir de régénérer la société en recherchant de nouveaux horizons, de diffuser des idées modernes, telles que " la fe en el progreso, el amor a la ciencia y a la libertad ${ }^{22}$, ne sauraient pourtant présager d'une victoire immédiate en Espagne.

De plus, la recherche d'un sens incite à la découverte des empreintes que les événements historiques et politiques ont laissées dans les civilisations occidentales. Aussi, Orloff doit-il sans cesse se situer par rapport à l'Histoire événementielle tout en analysant ses points d'ancrage les plus significatifs. À cette fin, il introduit à chaque étape des exposés documentaires, suivant les procédures habituelles des récits de voyage, dans le but de déceler les causes de son statut de perdant, mais surtout, de mettre en perspective les voies de changement. Vaincu en Russie, il se targue d'être vainqueur en Europe car il se projette dans une logique cosmopolite-issue des Lumières et des interprétations proposées par Kant, Goethe et Heine -, qu'il érige en bannière avant de se ranger sous celle de l'internationalisme du socialisme positif. Comme nous l'avions déjà signalé, le socialisme positif est un socialisme révisionniste visant à l'instauration de régimes républicains et démocratiques dans lesquels l'altruisme s'enracine en tant que dogme suprême de tous les citoyens.

Durant les deux années de vie intégrées dans le roman, le projet européen de Bark reste sous l'égide du pangermanisme au vu des conclusions de ses études de psychologie nationale. Ces conclusions mettent en exergue le génie

20. Ibid., p. 169.

21. Ibid., p. 229.

22. Ibid., p. 155. 
allemand mais aussi la complémentarité des peuples de l'Europe : "La sal ática, la pasión, la acción sont totalmente compatibles con la hondura, la serenidad y el rigor alemanes, por dar un ejemplo $»^{23}$.

L'hégémonie allemande, les succès diplomatiques de Bismarck, la décomposition des empires, l'émergence des nationalismes ainsi que l'éclosion des mouvements internationalistes catalysent l'utopie universaliste de Bark. Il prône, ainsi, un premier rapprochement hispano-allemand, puis, l'extension de ce rapprochement aux autres pays européens afin de mener à bien la construction d'une Europe sociale et démocratique moderne, autrement dit, la Confédération des États Unis de l'Europe. C'est précisément dans son roman que Bark pose les premiers jalons de la construction européenne. Si l'Europe a joué un rôle précurseur dans l'histoire de la civilisation, qu'elle soit pressentie comme l'espace idéal pour la Révolution Sociale reste un choix légitime.

Dans l'itinéraire européen d'Orloff, la découverte de l'altérité, l'insertion de digressions à visée idéologique, l'internationalisme, le système judiciaire, la libre-pensée, le panthéisme, l'anticléricalisme, la morale sociale, les réformes, la sociabilité, la condition féminine, l'éducation, la professionnalisation du journalisme, les fonctions sociales de l'art, le développement de la critique, constituent un échantillon des questions suggérées et des thèmes réfléchis de manière diffractée, réfractée ou anamorphosée dans le récit littéraire. Autant d'éléments qui façonnent le contenu socio-politique et culturel du roman et étayent la transmutation du vaincu en vainqueur, dans un présent se projetant dans un avenir dont Orloff souhaitait la réalisation au plus vite.

Enfin, dans le panorama espagnol, Los Vencidos d'Ernest Bark est considéré comme l'un des romans régénérationistes de la fin de siècle. Régénérationniste parce qu'il dit l'espoir, le changement, la réforme, la reconstruction, la victoire enfin, toutes choses qui pourraient relever du paradoxe si l'on s'en tient au titre. Alors, constat de la défaite ou cri salutaire? Seul le temps peut se porter juge. De nos jours, il est aisé d'en faire le constat. Les chimères d'Ernest Bark, du vaincu se voulant vainqueur, ne sont-elles pas des acquis dont nous bénéficions ? Certes. Mais pour l'histoire officielle, Ernest Bark demeure une histoire individuelle, celle d'un vaincu, d'un propagandiste et d'un passeur d'idées anonyme ayant sacrifié son existence, comme tant d'autres hommes, au service de la société.

23. «Para Bark, Francia representa la arrogancia, superficialidad y decadencia, simbolizadas todavía con la imagen de la monarquía de Luis XIV. La hegemonía italiana es específicamente artística por carecer de una política de talla. Sobre los ingleses pesa un acendrado materialismo mercantilista. Por último, los espańoles viven anclados en un pasado civilizador que no han sabido actualizar. Por consiguiente, el equilibrio de las tres esferas hegemónicas - política, espiritual y cultural - erigen el Imperio alemán en árbitro de la política mundial y pionero de la construcción de la fraternidad. Recordando a Fichte, Bark considera al pueblo alemán - individualista, cosmopolita, culto, sereno - especialmente apto para la lucha por la libertad y el progreso, lo cual refuerza su papel de guía internacional. En suma, Alemania ha de encaminar a los pueblos hacia la fraternidad propuesta como internacional o universal», Thion SorianoMollá, op. cit., p. 137. 